\title{
Influence of Resting-State Network on Lateralization of Functional Connectivity in Mesial Temporal Lobe Epilepsy
}

L. Su, J. An, Q. Ma, S. Qiu, and D. Hu O- $\equiv \triangle \square$

\begin{abstract}
BACKGROUND AND PURPOSE: Although most studies on epilepsy have focused on the epileptogenic zone, epilepsy is a system-level disease characterized by aberrant neuronal synchronization among groups of neurons. Increasingly, studies have indicated that mesial temporal lobe epilepsy may be a network-level disease; however, few investigations have examined resting-state functional connectivity of the entire brain, particularly in patients with mesial temporal lobe epilepsy and hippocampal sclerosis. This study primarily investigated whole-brain resting-state functional connectivity abnormality in patients with mesial temporal lobe epilepsy and right hippocampal sclerosis during the interictal period.
\end{abstract}

MATERIALS AND METHODS: We investigated resting-state functional connectivity of 21 patients with mesial temporal lobe epilepsy with right hippocampal sclerosis and 21 neurologically healthy controls. A multivariate pattern analysis was used to identify the functional connections that most clearly differentiated patients with mesial temporal lobe epilepsy with right hippocampal sclerosis from controls.

RESULTS: Discriminative analysis of functional connections indicated that the patients with mesial temporal lobe epilepsy with right hippocampal sclerosis exhibited decreased resting-state functional connectivity within the right hemisphere and increased resting-state functional connectivity within the left hemisphere. Resting-state network analysis suggested that the internetwork connections typically obey the hemispheric lateralization trend and most of the functional connections that disturb the lateralization trend are the intranetwork ones.

CONCLUSIONS: The current findings suggest that weakening of the resting-state functional connectivity associated with the right hemisphere appears to strengthen resting-state functional connectivity on the contralateral side, which may be related to the seizureinduced damage and underlying compensatory mechanisms. Resting-state network-based analysis indicated that the compensatory mechanism among different resting-state networks may disturb the hemispheric lateralization.

ABBREVIATIONS: $\mathrm{DMN}=$ default-mode network; $\mathrm{FC}=$ functional connectivity or connection; $\mathrm{HS}=$ hippocampal sclerosis; $\mathrm{mTLE}=$ mesial temporal lobe epilepsy; R-mTLE = mesial temporal lobe epilepsy with right hippocampal sclerosis; RS = resting-state; RSN = resting-state network; TLE = temporal lobe epilepsy

U p to $0.1 \%$ of the human population worldwide has temporal lobe epilepsy (TLE), and 60\%-70\% of these cases are classified as mesial temporal lobe epilepsy (mTLE). ${ }^{1}$ mTLE is a drug-refractory form of human epilepsy that is typically char-

Received October 1, 2014; accepted after revision January 25, 2015.

From the College of Mechatronics and Automation (L.S., Q.M., D.H.), National University of Defense Technology, Changsha, Hunan, People's Republic of China; Department of Medical Imaging (J.A., S.Q.), First Affiliated Hospital of Guangzhou University of Chinese Medicine, Guangzhou, Guangdong, People's Republic of China; and Department of Information Engineering (L.S.), Officers College of Chinese Armed Police Force, Chengdu, Sichuan, China.

This study was supported by the National Basic Research Program of China (2011CB707802, 81271389, 81471251, 91420302, and 61420106001).

Drs Longfei Su and Jie An contributed equally to this work.

Please address correspondence to Dewen Hu, PhD, No. 109, Deya Rd, Kaifu District, Changsha City, Hunan Province, People's Republic of China; e-mail: dwhu@nudt. edu.cn; and Shijun Qiu, PhD, First Affiliated Hospital of Guangzhou University of

acterized by hippocampal sclerosis (HS). Surgical intervention can prevent temporal lobe seizure recurrence in patients with mTLE. $^{2}$ Aberrant neuronal synchronization is believed to be as important as abnormal excitability with respect to epileptic seizure occurrences, ${ }^{3,4}$ and resting-state functional connectivity (RS-FC) analysis is an effective approach for examining neural synchronization.

Debilitating mTLE seizures are believed to originate primarily from specific anatomic divisions of the temporal lobe. ${ }^{2}$

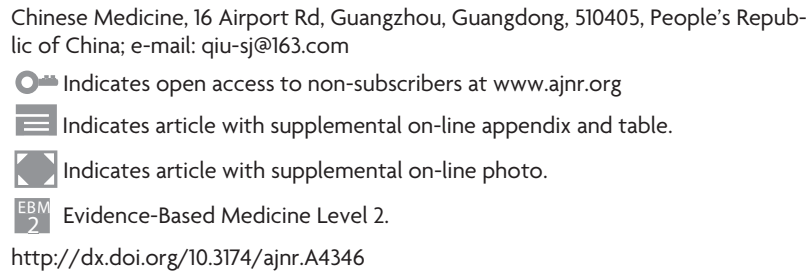


Demographic and clinical data

\begin{tabular}{lccc}
\hline & \multicolumn{2}{c}{ Mean (Range) } & \\
\cline { 2 - 3 } & \multicolumn{1}{c}{ R-mTLE } & Control & P Value \\
\hline Sample size & 21 & 21 & - \\
Sex (M/F) & $9: 12$ & $9: 12$ & $1.000^{\mathrm{a}}$ \\
Age (yr) & $28.5 \pm 7.9(18-43)$ & $25.1 \pm 5.7(17-37)$ & $.187^{\mathrm{b}}$ \\
Education (yr) & $11.6 \pm 2.3(9-16)$ & $11.5 \pm 2.7(6-16)$ & $.891^{\mathrm{b}}$ \\
Onset (yr) & $15.7 \pm 9.8(2-34)$ & - & - \\
Duration (yr) & $12.9 \pm 7.4(3-33)$ & - & - \\
\hline
\end{tabular}

a Pearson $\chi^{2}$ test.

${ }^{\mathrm{b}}$ Two-sample $t$ test.

However, investigations involving large-scale network analysis have challenged this traditional conceptualization. ${ }^{3,5-7}$ Furthermore, several studies have reported an mTLE-related decrease in basal RS-FC in the epileptogenic hemisphere in brains of patients with mTLE, accompanied by contralateral compensatory mechanisms. ${ }^{8,9}$

Many mTLE studies have focused on the epileptogenic zone, and most analyses that have investigated regions outside the hippocampus have focused on structural imaging technology. ${ }^{1,10,11}$ In contrast, few whole-brain functional network analyses of mTLE have been conducted. Structural MRI or electroencephalography or both incompletely measure temporal changes during the disease process, ${ }^{5}$ while resting-state fMRI takes serial images during a time period that can capture the dynamic and evolving changes related to epilepsy. ${ }^{12}$ The RS-FC derived from the fMRI images reflects functional aberrations and offers a network perspective on the psychiatric and cognitive complications of mTLE. ${ }^{7,13}$ We hypothesized that mesial temporal lobe epilepsy with right hippocampal sclerosis (R-mTLE) is a functional disease involving disturbances of RS-FC over the entire brain rather than a local disease that is confined to the temporal lobe. To test this hypothesis, we applied the multivariate pattern analysis method in this study. ${ }^{14}$

\section{MATERIALS AND METHODS Participants}

We studied 21 consecutive right-handed patients with R-mTLE who underwent presurgical evaluation at Guangdong 999 Brain Hospital. Diagnoses of R-mTLE and the lateralization of the seizure foci of this disease were determined via comprehensive evaluations that included examinations of the patients' detailed medical histories, video-electroencephalography telemetry, and neuroimaging. The presence of abnormally elevated T2 fluid-attenuated inversion recovery signals in the hippocampus was used as the diagnostic criterion for HS. In all patients, the HS site corresponded to the epileptogenic site. None of the patients with R-mTLE had mass lesions (tumor, vascular malformation, or malformations of cortical development) or traumatic brain injury. HS was detected in all patients following qualitative histopathologic analysis. ${ }^{15}$ Twenty-one healthy right-handed subjects were recruited as controls; these controls were matched to the examined patients with R-mTLE with respect to age, sex, and years of education (Table). All controls were medically healthy and free of any neurologic or psychiatric disorders at the time of the study.

\section{Standard Protocol Approvals, Registrations, and Patient Consents}

This study was approved by the Research Ethics Review Board of the Institute of Mental Health of Southern Medical University. Informed consent was obtained from each subject.

\section{Imaging Protocol}

During the experiments, subjects were instructed to keep their eyes closed, relax, and remain awake. Subjects were asked not to perform any specific cognitive exercises. After each session, the subjects were asked whether they had fallen asleep during the preceding session, and all subjects confirmed that they had remained awake throughout the experiment. Functional MR images were acquired by using an Intera 1.5T MR scanner (Philips Healthcare, Best, the Netherlands) with a gradient-echo EPI sequence. We used the following imaging parameters: TR/TE = $3000 / 50 \mathrm{~ms}$; thickness $/$ gap $=4.5 / 0 \mathrm{~mm} ; \mathrm{FOV}=230 \times 230 \mathrm{~cm}$; flip angle $=90^{\circ}$; matrix $=128 \times 128$; sections $=31$. The duration of each functional resting-state session was approximately 8 minutes, and 160 volumes were obtained for each patient.

\section{Data Preprocessing}

Data preprocessing was performed by using the Statistical Parametric Mapping software package (SPM8; http://www.fil.ion. ucl.ac.uk/spm/software/spm12). For each subject, the first 10 volumes of scanning data were discarded to reduce magnetic saturation effects. The remaining 150 volumes of data were corrected by registering and reslicing for head motion. Subsequently, these volumes were normalized to standard echo-planar imaging templates in Montreal Neurological Institute space. The resulting images were spatially smoothed with a Gaussian filter with an 8-mm full width at half maximum kernel, detrended to remove linear trends, and temporally filtered with a Chebyshev bandpass filter $(0.01-0.08 \mathrm{~Hz})$ to reduce artifacts caused by respiration or cardiac action. All fMRI volumes were registered to a Montreal Neurological Institute template before further time-series extraction for ROIs. The Montreal Neurological Institute coordinates for the ROIs in this template are shown in the On-line Table. Each regional mean time-series was further corrected for the effects of WM, CSF, and head movement by regression on the time-series of $\mathrm{WM}, \mathrm{CSF}$ signal and translations, and rotations of the head estimated in the course of initial movement correction by image realignment. The residuals of these regressions constituted the set of regional mean time-series used for functional connectivity analysis. $^{16}$

The center coordinates were defined as the areas of peak activity identified in 5 meta-analyses that focused on error processing, default-mode network (DMN), memory, language, and sensorimotor functions. Although these functional networks were identified in task-related studies, they have been confirmed by many previous resting-state fMRI studies and were further used as resting-state networks (RSNs). ${ }^{17-20}$ Inevitably, using this pre-existing localization of ROIs can introduce bias; however, using a priori ROIs also offers a substantial increase in power. ${ }^{4}$

We used the Pearson correlation coefficient to evaluate the functional connectivity between each pair of ROIs. This approach allowed us to obtain symmetric $160 \times 160$ matrices that captured 
the resting-state functional network activity of each subject. After removing the 160 diagonal elements of each functional connectivity matrix, we extracted the upper triangle elements of these connection matrices as classification features; therefore, the feature space for classification was spanned by the remaining $(160 \times$ 159) $/ 2=12,720$ dimensional feature vectors. In this article, the functional connections (FCs) for classification are referred to as "features."

Alternatively, an unsampled version of the automatic anatomic labeling template was introduced for ROI definition, which segmented the cerebrum into 600 ROIs. Further analysis by using this template and selection of ROI radii are displayed in "ROI Definition for the Entire Brain” in the On-Line Appendix.

Identification of Features with High Discriminative Power The support vector machine recursive feature elimination algorithm was originally proposed for gene selection ${ }^{21}$ and has been applied in fMRI studies for the identification of multiple active voxels. ${ }^{22}$ The support vector machine recursive feature elimination algorithm combines the support vector machine and recursive feature elimination approaches to produce a multivariate feature-selection algorithm. In the support vector machine classification procedure, all samples are categorized into 2 parts. One portion of the samples with class labels was used to train the classifier, called "training samples." The training samples can be used to identify the parameters of the classifier. All training samples constructed the training set. The other portion of the samples without class labels was used to test the effectiveness of the classifier called "testing samples." The classifier can predict the class labels of the testing samples. The support vector machine classification was applied to the training set, and the discriminative weight $w\left(f_{\mathrm{i}}\right)$ of feature $f_{\mathrm{i}}$ was obtained for each training sample. The scoring function was defined as follows:

$$
\operatorname{Score}\left(f_{\mathrm{i}}\right)=\frac{\sum_{k=1}^{n}\left|w_{\mathrm{k}}\left(f_{\mathrm{i}}\right)\right|}{n}
$$

In the equation above, $f_{\mathrm{i}}$ represents the $i$ th feature, $w_{\mathrm{k}}\left(f_{\mathrm{i}}\right)$ represents the discriminative weight of feature $f_{\mathrm{i}}$ in the $k$ th sample, and $n$ is the number of training samples. The features $f_{\mathrm{i}}, i=1,2, \cdots, n$ were then ranked by $\operatorname{Score}\left(f_{\mathrm{i}}\right), i=1,2, \cdots, n$, and the feature with the smallest score was eliminated. This procedure was repeated on the retained features until all features were eliminated. To accelerate the computational process, we eliminated the half of the remaining features with the smallest scores in each iteration of the algorithm.

The details of identification of discriminative FCs are displayed in "Cross-Validation and Consensus Functional Connections" in the On-line Appendix.

\section{Support Vector Classification and Performance Evaluation}

Support Vector Classification. After the dataset of features had been prepared, linear support vector machines were used to solve the classification problem. ${ }^{23}$ All pattern analyses were implemented by using the LIBSVM software package (http://www.csie.ntu.edu.tw/ cjlin/libsvm/).

Performance Evaluation. Based on the cross-validation results, the performance of a classifier was quantified in terms of the generalization rate, sensitivity, and specificity. ${ }^{24}$ Notably, "sensitivity" represents the proportion of patients who were correctly classified, and "specificity" represents the proportion of controls who were correctly classified. The overall proportion of correctly classified samples was evaluated by using the generalization rate.

\section{RESULTS}

\section{Classification Results}

The network analysis was based on the classification results. The classification accuracy rates relative to the number of selected FCs are indicated in Fig 1. Classification accuracies (expressed in terms of the generalization rate) of $>90 \%$ were achieved in classification approaches that used relatively few (approximately $10-$ 50) FCs.

In particular, when the first 8 FCs were used, a sensitivity of $95.2 \%$ and a specificity of $95.2 \%$ were obtained (only 1 patient and 1 control participant were not successfully identified). Furthermore, if the support vector machine classification boundary was replaced with the optimal classification boundary, a sensitivity of $95.2 \%$ and a specificity of $100 \%$ were obtained (only 1 patient was not successfully identified), which are reflected in Fig $2 B$. When the first 23 connections were used, a sensitivity of $90.5 \%$ and a specificity of $100 \%$ were obtained (only 2 patients were not successfully classified). Furthermore, if the optimal classification boundary was used, a sensitivity of $100 \%$ and a specificity of $100 \%$ were obtained (all subjects were correctly classified), which were also reflected by the receiver operating characteristic in Fig 2D.

Using the generalization rate as the applicable statistic, we determined the permutation distribution of estimates, which are shown in Fig $2 A,-C$; the results indicated that the classifier learned the relationship between the data and the labels with a $<.0001$ probability of being incorrect.

Because a leave-one-out cross-validation approach was used, the results represent estimations of the classification accuracy for the scanning results for a new subject; thus, these findings have a direct diagnostic relevance. The receiver operating characteristic curves of the classifiers, which were determined by using a leaveone-out cross-validation approach, are depicted in Fig $2 B,-D$. The area under the receiver operating characteristic curve for the classification method was $99.6 \%$ when 8 FCs were used and $100 \%$ when 23 FCs were used.

In the present study, each ROI was defined as a sphere with a given center coordinate and a radius of $5 \mathrm{~mm}$. Radii of 4 and 7.5 $\mathrm{mm}$ were also used for ROI definitions, but the classification results were not better than those of the radius of $5 \mathrm{~mm}$, which are displayed in On-line Fig 1.

\section{Functional Connectivity Changes}

The RS-FC analysis in this study primarily focused on R-mTLErelated alterations in the strengths of FCs. The first finding of this study was the hemispheric lateralization of the changes in RS-FC. In summary, weaker connections in patients with R-mTLE relative to healthy controls were mainly located in the right hemi- 


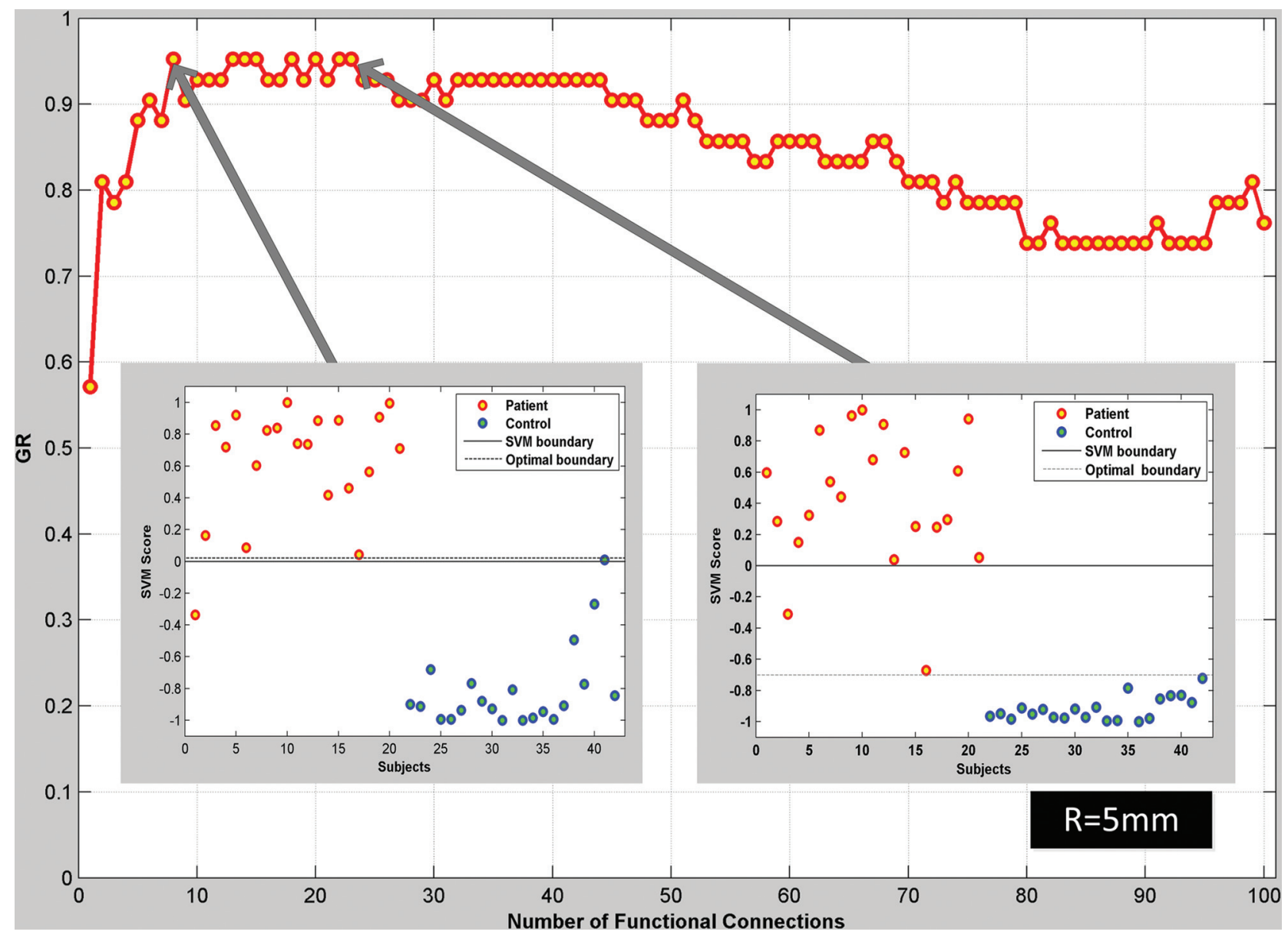

FIG 1. Classification results for patients with R-mTLE and healthy controls via leave-one-out cross-validation. The x-axis indicates the number of connections involved in the classification; the $y$-axis indicates classification accuracy (as represented by the generalization rate). The subplot illustrates the prediction results of all subjects with the highest accuracy, which used the first 8 and the first 23 most discriminative connections.

sphere (Fig 3A), and stronger connections in patients with RmTLE relative to healthy controls were mainly distributed in the left hemisphere (Fig 3B).

These altered FCs may be categorized as either intranetwork connections, connecting nodes within the same functional network; or internetwork connections, connecting nodes from 2 different functional networks. The second finding of this study was that compared with healthy controls, patients with R-mTLE showed a different internetwork pattern compared with the intranetwork connectivity changes.

Most intranetwork FCs were weaker, while more internetwork FCs were stronger in the patients with R-mTLE compared with controls (Fig $4 A,-B$ ).

\section{DISCUSSION}

In this study, we sought to validate the hypothesis that R-mTLE is a disease with RSN disturbances. Multivariate pattern analysis results indicated that R-mTLE-related changes in functional connectivity predominantly follow this pattern: Weaker connections were primarily distributed in the right hemisphere, while most of the stronger connections were in the left hemisphere. Additional RSN analysis demonstrated that most of the altered FCs-not strictly associated with hemispheric lateralization-were intranetwork FCs; most of the FCs — which primarily obeyed the hemi- spheric lateralization-were internetwork FCs. Specifically, weaker connections were localized within the DMN, cinguloopercular network, and frontoparietal network, whereas stronger connections were localized within the sensorimotor network.

\section{RSN Analysis of Brain Regions beyond the Hippocampus}

To validate the hypothesis that mTLE is a network disease, some studies have investigated abnormalities in brain regions other than the hippocampus. ${ }^{1,6,10,11,25,26}$ In addition, the support vector classification accuracy of structural MRI and DTI datasets that exclude the hippocampus can reach approximately $90 \% .{ }^{10}$ Given that resting-state fMRI can capture dynamic and evolving changes related to epilepsy, ${ }^{12}$ we applied RS-FC on the basis of fMRI data. Although the hippocampus is particularly important for the identification of R-mTLE, this study sought to demonstrate that RmTLE is a disease affecting the RSN of the entire brain rather than a local disease that is limited to hippocampal aberrations. The ROI template used in this investigation, which facilitated the RSN analysis and excluded the hippocampus, achieved classification accuracies up to $95 \%$. This result provides important evidence that R-mTLE is a network disease characterized by functional aberrations distributed across the entire brain. In addition, we defined the epileptogenic zone through the use of a 2-sample $t$ test, which compared patients with R-mTLE with healthy con- 

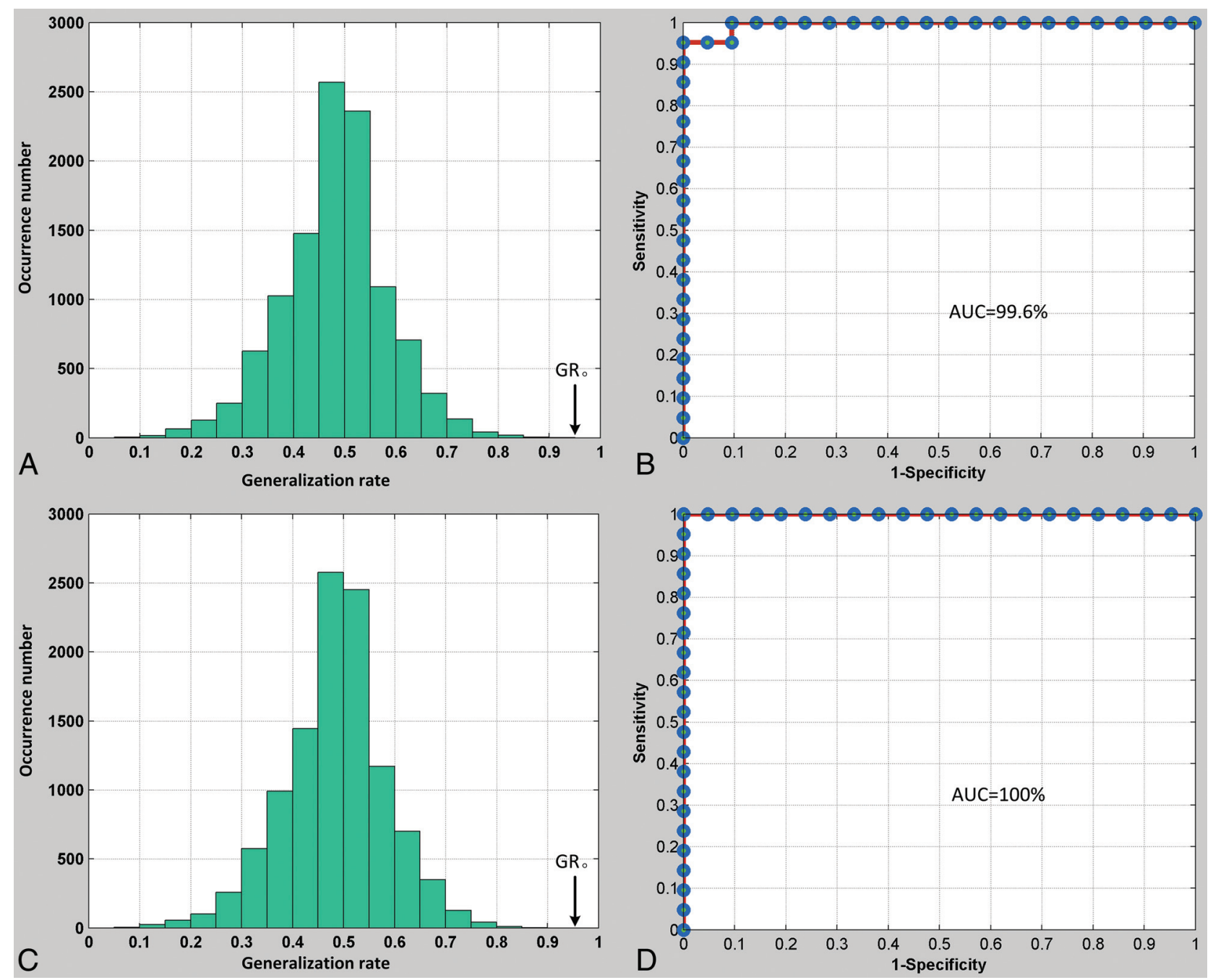

FIG 2. Classification evaluation. A, The permutation distribution of estimates produced by the linear support vector machine classifier (with 10,000 repetitions) if the first 8 most discriminating features are used. The $x$ - and $y$-axes indicate the generalization rate and occurrence number; $\mathrm{GR}_{0}$, is the generalization rate obtained by the classifier trained on the actual class labels. Using the generalization rate as the test statistic, this figure demonstrates that the classifier learned the relationship between the data and the labels with a probability of being incorrect of $<.0001$. $B$, Receiver operating characteristic curves indicate the overall classification performance of the functional connectivity-based classification of patients with R-mTLE and healthy controls. The area under the receiver operating characteristic curve (AUC) was $99.6 \%$ when the first 8 connections were used for classification. $C$, The permutation distribution of estimates produced by the linear support vector machine classifier (with 10,000 repetitions) if the first 23 most discriminating features are used. $D$, Receiver operating characteristic curves indicate the overall classification performance of the functional connectivity-based classification of patients with R-mTLE and healthy controls. The area under the receiver operating characteristic curve was $100 \%$ when the first 23 connections were involved in the classification.

trols, and this zone was added to the ROI template. Whole-brain functional connectivity was determined on the basis of the $160+$ 1 ROIs mentioned above. The resulting classification accuracy was not improved, and the identified discriminative connections were identical to the results presented in this article.

\section{Identification of FCs}

From a functional integration perspective, RS-FC analysis exhibits advantages relative to other modalities, ${ }^{27}$ particularly when multivariate pattern analysis methods are used. ${ }^{28}$ The support vector machine recursive feature elimination method can predict group membership at an individual subject level, and the results obtained by using this method may be clinically useful ${ }^{29}$ because the results can include unique information that may be overlooked by univariate voxel-based morphometry approaches. ${ }^{30,31}$ As indicated in Fig 1, maximal classification accuracy (generaliza- tion rate $=95.2 \%$, area under the receiver operating characteristic curve $=100 \%$ ) was obtained by using only 23 features. We assigned connection strengths on the basis of the occurrence of these connections in the leave-one-out cross-validation results (Fig 3). As the number of features increased beyond 23, the classification accuracy generally decreased. This result suggests that only a few of the 12,720 examined connections were highly discriminative.

\section{Different R-mTLE-Related RS-FC Changes in the Epileptogenic and Contralateral Sides of the Brain}

An initial finding of this study was that changes in RS-FC in patients with R-mTLE demonstrate hemispheric lateralization. Weaker FCs in patients with R-mTLE were primarily distributed in the right hemisphere (Fig $3 A$ ). However, most of the stronger FCs in the patients with R-mTLE were located in the left hemi- 

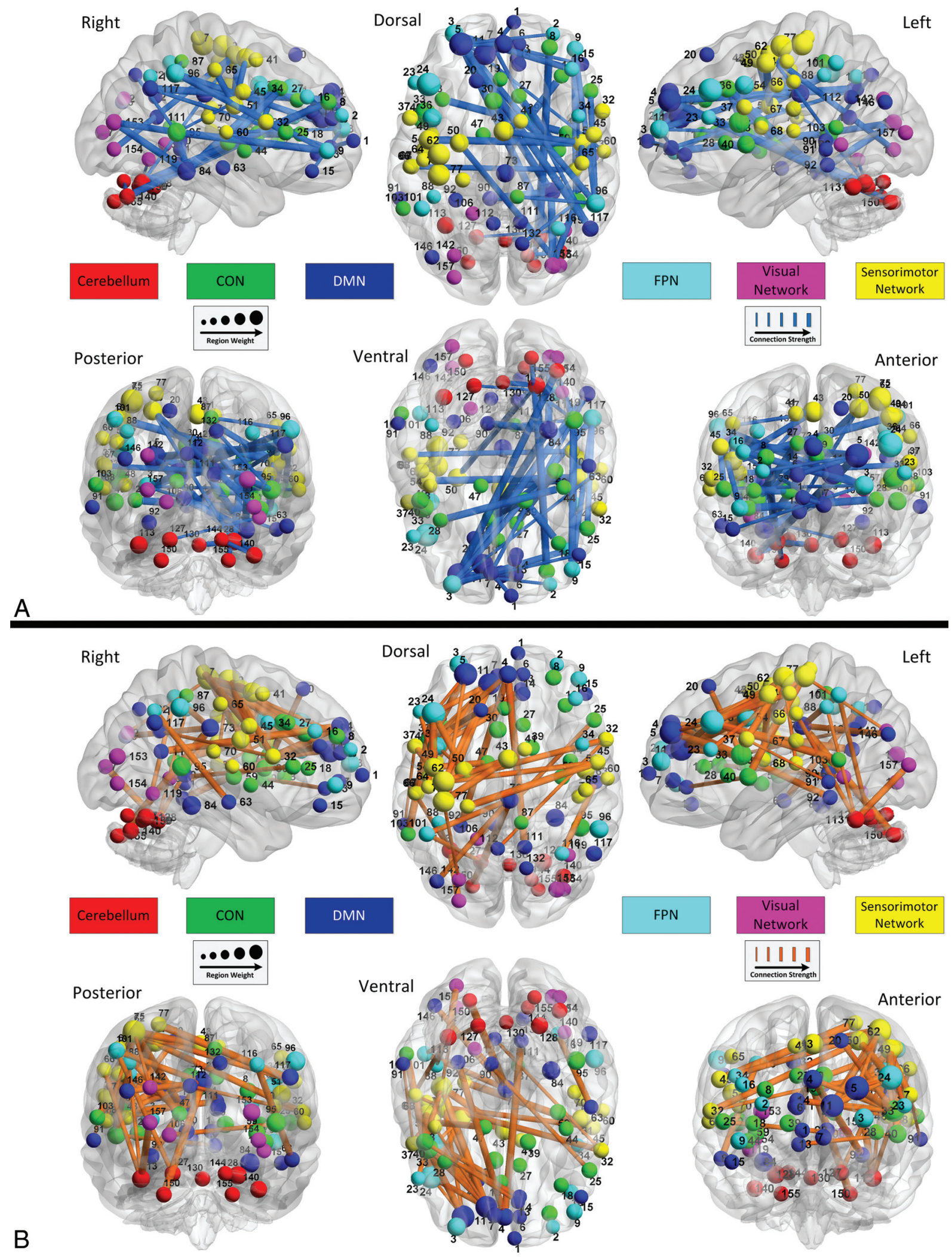

FIG 3. Region weights and connection strengths categorized by hemisphere. The connections are displayed in a surface rendering of a human brain. The thicknesses of the consensus connections in the leave-one-out cross-validation are scaled by their strengths (which were the normalized occurrences of the first 23 connections during all iterations of the leave-one-out cross-validation). A, Connections with lower strengths in patients with R-mTLE than in controls are depicted in light blue. B, Connections with greater strengths in patients with R-mTLE than in controls are displayed in orange. The ROls related to the selected consensus connections are also scaled by their weights (calculated as the sum of the weights of all connections to and from the ROI) and are displayed. The ROIs are color-coded by functional network (cerebellum, red; cingulo-opercular network, green; DMN, blue; frontoparietal network, cyan; visual network, rose; and sensorimotor network, yellow). The numeric labels for the ROIs in this figure are provided in the On-line Table. 

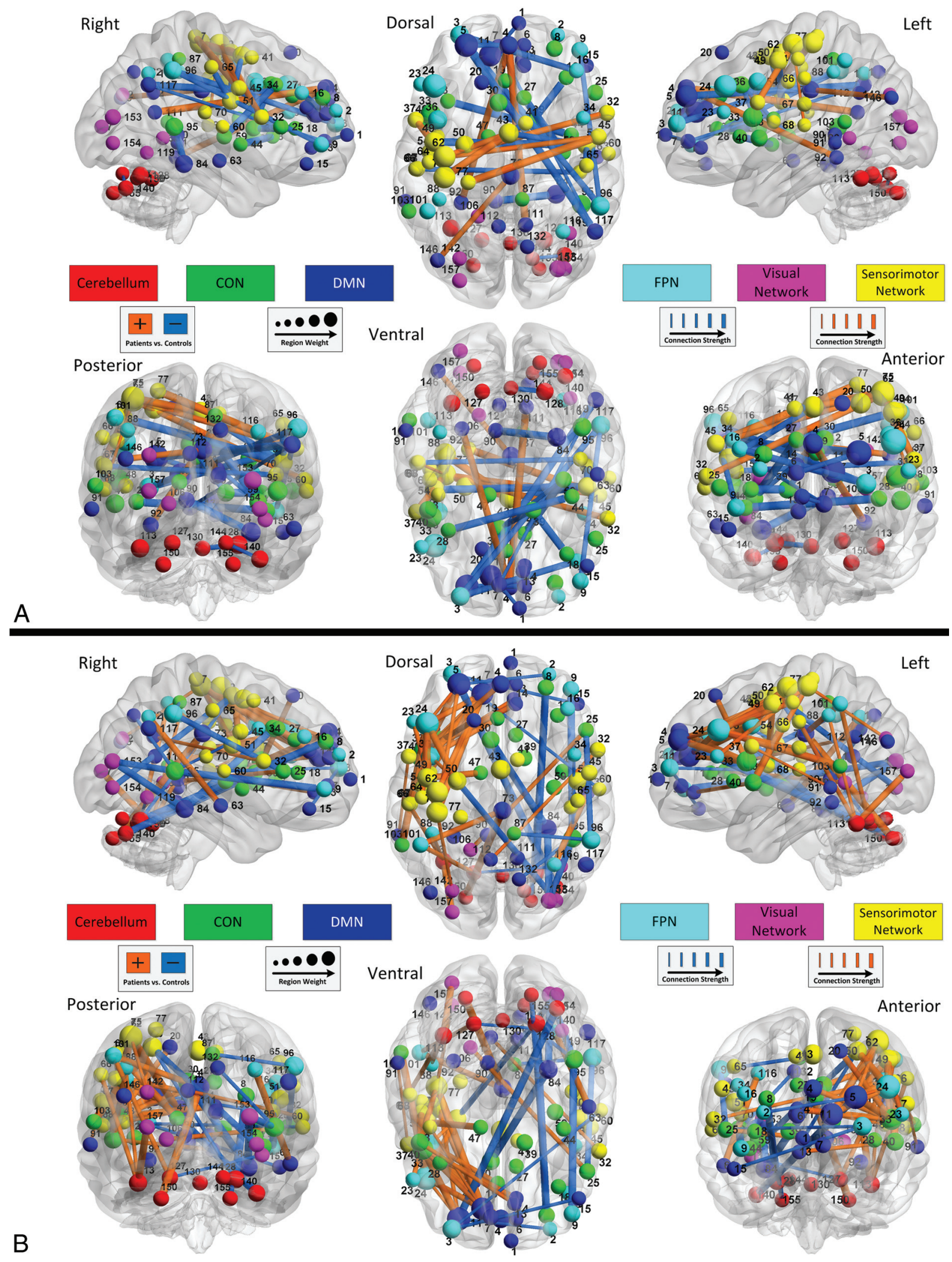

FIG 4. Region weights and connection strengths viewed from inter- and intranetwork perspectives. The connections are displayed in a surface rendering of a human brain. The thicknesses of the consensus connections in the leave-one-out cross-validation are scaled by their strengths (which were the normalized occurrences of the first 23 connections during all iterations of the leave-one-out cross-validation). $A$, Internetwork connections. B, Intranetwork connections. Connections with greater strengths in patients with R-mTLE than in controls are displayed in orange. Connections with lower strengths in patients with R-mTLE than in controls are depicted in light blue. The ROIs related to the selected consensus connections are also scaled by their weights (calculated as the sum of the weights of all connections to and from the ROI of interest) and are displayed. The ROls are color-coded as in Fig 3. 
sphere (Fig 3B). Previous studies have attributed the lateralization of RS-FC in mTLE to compensatory mechanisms in the human brain. ${ }^{8,9}$ However, this conclusion was derived from RS-FC analyses that were restricted to the hippocampus and several other brain regions that are closely related to mTLE. Furthermore, only a small sample of patients with mTLE and even fewer patients with R-mTLE were examined in the prior studies. Our study provides further support for the hypothesis that patients with mTLE demonstrate decreased functional connectivity in the epileptogenic sides of their brains but exhibit contralateral compensatory mechanisms. First, our study focused on brain regions outside the hippocampus, thereby suggesting that the pathophysiology may be more widely distributed than previously recognized,. The weaker RS-FC in the contralateral hemisphere suggests a compensatory mechanism that involves the entire brain. Second, previous studies have examined only a small number of patients with RmTLE. Relative to these prior studies, our investigation included a larger number of participants with R-mTLE.

Further evidence is needed to validate the hemispheric lateralization of the RS-FC in mTLE. To validate this hemispheric lateralization, we also investigated GM and WM concentrations and the GM and WM presenting with similar hemispheric lateralizations, which are detailed in "Voxel-Based Morphometric Analysis" in the Appendix and On-line Fig 2.

\section{RSN Analysis of R-mTLE}

The second finding of the current study is that the intranetwork FCs were weakened, while the internetwork RS-FC was increased. It is generally believed that the decrease in RS-FC reflects an impairment in the functional network related to the corresponding RSN, while an increased RS-FC may indicate enhanced function due to the compensatory mechanism. ${ }^{32,33}$ Furthermore, most of the FCs that did not conform to the observed pattern of hemispheric lateralization were intranetwork FCs. In contrast, most internetwork FCs followed the observed laterality patterns. This result may indicate that as functional units, the RSN and the hemisphere influence each other.

Intranetwork FCs that were weakened in patients with R-mTLE relative to control subjects were mainly localized to the DMN cingulo-opercular network and frontoparietal network. In contrast, intranetwork FCs that were strengthened in patients with R-mTLE relative to control subjects were localized to the sensorimotor network. The DMN is characterized by task-induced deactivation, which is essential for maintaining baseline levels of brain activities related to self-awareness, episodic memory, and environmental monitoring. ${ }^{34}$ In recent years, the DMN has been reported to be decreased in RS-FC and has attracted considerable attention in mTLE research. ${ }^{35-37}$ Previous studies also found that the RS-FC in the frontoparietal network was decreased in mTLE. ${ }^{37,38}$ The strength of most intranetwork connections in the DMN, cingulo-opercular network, and frontoparietal network was reduced in patients with R-mTLE relative to healthy controls in this study, consistent with a previous independent component analysis-based study. ${ }^{38}$ This result may indicate that R-mTLE produces disturbances in executive control functions for the DMN, cingulo-opercular network, and fronto-parietal network, which are believed to be closely related to executive control tasks. ${ }^{39-41}$ Patients with mTLE demonstrate apparent executive deficits. ${ }^{42-44}$ In previous studies, the sensorimotor network had abnormal RS-FC and the patients with mTLE displayed cognitive impairments. ${ }^{38,43,45}$ The increased RS-FC in the sensorimotor network in the current study may be a compensatory mechanism between the left and the right sensorimotor cortex as shown in Fig $3 B$.

In contrast, internetwork connections between the aforementioned RSNs were generally stronger in patients with R-mTLE than in control subjects. This phenomenon may have been produced by compensatory mechanisms. The increased RS-FC strength reflects increased spontaneous synchronization among brain regions, and previous studies have attributed increased RS-FC in mTLE to underlying compensatory mechanisms. ${ }^{35} \mathrm{Sev}$ eral age-related studies have reported that the weakening of shortrange connectivity and the strengthening of long-range connectivity during aging are driven by functional segregation and integration, respectively. ${ }^{14,46,47}$ In addition, a published article reported that patients with mTLE demonstrated decreased local functional connectivity and increased intrahemispheric functional connectivity. ${ }^{33}$ However, the results obtained in our study suggest that R-mTLE induces impairments in specific functional networks and that the functional networks become more integrated to compensate for deficits caused by these impairments. We propose that the compensatory mechanism involves interactions between distinct functional units and cannot simply be assessed in terms of anatomic distance.

Finally, comparing Figs 3 and 4, we found that most intranetwork FCs were interhemispheric FCs, which indicates that the compensatory mechanism among different RSNs may disturb the hemispheric lateralization.

\section{Limitations and Future Work}

There were several limitations in our study. First, it examined a small sample. Our findings must be replicated with larger datasets before the findings of this investigation can be broadly generalized to patient populations with R-mTLE. Second, mesial temporal lobe epilepsy with left HS was not considered in our study. In future studies, we plan to address these limitations by conducting multimodal network analyses to investigate patients with mesial temporal lobe epilepsy with left HS and R-mTLE.

\section{CONCLUSIONS}

Based on the classification results, we found that compared with connections of the healthy controls, weakened connections of the patients with R-mTLE were primarily distributed in the right hemisphere, whereas the majority of strengthened connections were located in the left hemisphere. Additional RSN analyses demonstrated that most of the altered FCs-not strictly associated with hemispheric laterality — were intranetwork FCs; most of the FCs — which tended to obey the hemispheric laterality — were internetwork FCs.

\section{ACKNOWLEDGMENTS}

The authors thank the anonymous reviewers for their constructive suggestions and Ann M. Hermundstad for providing us the unsampled version of the automatic anatomic labeling template. 
Disclosures: Longfei Su—RELATED: Grant: National Natural Science Foundation of China, ${ }^{*}$ Comments: National Basic Research Program of China (2011CB707802) and the National Natural Science Foundation of China (61003202, 61005084). *Money paid to the institution.

\section{REFERENCES}

1. Mueller SG, Laxer KD, Barakos J, et al. Widespread neocortical abnormalities in temporal lobe epilepsy with and without mesial sclerosis. Neuroimage 2009;46:353-59

2. Engel J Jr. Mesial temporal lobe epilepsy: what have we learned? Neuroscientist 2001;7:340-52

3. Spencer SS. Neural networks in human epilepsy: evidence of and applications for treatment. Epilepsia 2002;43:219-27

4. Engel J, Thompson PM, Stern JM, et al. Connectomics and epilepsy. Curr Opin Neurol 2013;26:186-94

5. Maccotta L, He BJ, Snyder AZ, et al. Impaired and facilitated functional networks in temporal lobe epilepsy. Neuroimage Clin 2013; 2:862-72

6. Voets NL, Beckmann CF, Cole DM, et al. Structural substrates for resting network disruption in temporal lobe epilepsy. Brain 2012;135:2350-57

7. Cataldi M, Avoli M, de Villers-Sidani E. Resting state networks in temporal lobe epilepsy. Epilepsia 2013;54:2048-59

8. Bettus G, Bartolomei F, Confort-Gouny S, et al. Role of resting state functional connectivity MRI in presurgical investigation of mesial temporal lobe epilepsy. J Neurol Neurosurg Psychiatry 2010;81: 1147-54

9. Bettus G, Guedj E, Joyeux F, et al. Decreased basal fMRI functional connectivity in epileptogenic networks and contralateral compensatory mechanisms. Hum Brain Mapp 2009;30:1580-91

10. Focke NK, Yogarajah M, Symms MR, et al. Automated MR image classification in temporal lobe epilepsy. Neuroimage 2012;59:356-62

11. Mueller SG, Laxer KD, Cashdollar N, et al. Voxel-based optimized morphometry (VBM) of gray and white matter in temporal lobe epilepsy (TLE) with and without mesial temporal sclerosis. Epilepsia 2006;47:900-07

12. Richardson M. Current themes in neuroimaging of epilepsy: brain networks, dynamic phenomena, and clinical relevance. Clin Neurophysiol 2010;121:1153-75

13. Zhang Z, Liao W, Chen $\mathrm{H}$, et al. Altered functional-structural coupling of large-scale brain networks in idiopathic generalized epilepsy. Brain 2011;134:2912-28

14. Dosenbach NU, Nardos B, Cohen AL, et al. Prediction of individual brain maturity using fMRI. Science 2010;329:1358-61

15. Meencke H, Veith G. Hippocampal sclerosis in epilepsy. In: Lüders H, ed. Epilepsy Surgery. New York: Raven; 1991:705-15

16. Achard S, Salvador R, Whitcher B, et al. A resilient, low-frequency, small-world human brain functional network with highly connected association cortical hubs. J Neurosci 2006;26:63-72

17. Lee MH, Smyser CD, Shimony JS. Resting-state fMRI: a review of methods and clinical applications. AJNR Am J Neuroradiol 2013; 34:1866-72

18. van den Heuvel MP, Hulshoff Pol HE. Exploring the brain network: a review on resting-state fMRI functional connectivity. Eur Neuropsychopharmacol 2010;20:519-34

19. Repovs G, Csernansky JG, Barch DM. Brain network connectivity in individuals with schizophrenia and their siblings. Biol Psychiatry 2011;69:967-73

20. Tedeschi G, Esposito F. Neuronal networks observed with resting state functional magnetic resonance imaging in clinical populations. In: Bright P. Neuroimaging Cognitive and Clinical Neuroscience. North Logan: InTech; 2012:3

21. Guyon I. Gene selection for cancer classification using support vector machines. Mach Learn 2002;46:389-422

22. De Martino FD, Valente G, Staeren N, et al. Combining multivariate voxel selection and support vector machines for mapping and classification of fMRI spatial patterns. Neuroimage 2008;43:44-58
23. Vapnik V. The Nature of Statistical Learning Theory. New York: Springer-Verlag; 1995

24. Fawcett T. An introduction to ROC analysis. Pattern Recognition Letters 2006;27:861-74

25. Focke NK, Yogarajah M, Bonelli SB, et al. Voxel-based diffusion tensor imaging in patients with mesial temporal lobe epilepsy and hippocampal sclerosis. Neuroimage 2008;40:728-37

26. Bernhardt BC, Chen Z, He Y, et al. Graph-theoretical analysis reveals disrupted small-world organization of cortical thickness correlation networks in temporal lobe epilepsy. Cereb Cortex 2011;21:2147-57

27. Friston KJ, Frith CD. Schizophrenia: a disconnection syndrome? Clin Neurosci 1995;3:89-97

28. Shen H, Wang L, Liu Y, et al. Discriminative analysis of resting-state functional connectivity patterns of schizophrenia using low dimensional embedding of fMRI. Neuroimage 2010;49:3110-21

29. Gong Q, Wu Q, Scarpazz C, et al. Prognostic prediction of therapeutic response in depression using high-field MR imaging. Neuroimage 2011;55:1497-503

30. Haynes JD, Rees G. Decoding mental states from brain activity in humans. Nat Rev Neurosci 2006;7:523-34

31. Su L, Wang L, Chen F, et al. Sparse representation of brain aging: extracting covariance patterns from structural MRI. PloS One 2012;7:e36147

32. Zhang Z, Lu G, Zhong Y, et al. Impaired attention network in temporal lobe epilepsy: a resting FMRI study. Neurosci Lett 2009;458: 97-101

33. Greicius MD, Flores BH, Menon V, et al. Resting-state functional connectivity in major depression: abnormally increased contributions from subgenual cingulate cortex and thalamus. Biol Psychiatry 2007;62:429-37

34. Buckner RL, Andrews-Hanna JR, Schacter DL. The brain's default network: anatomy, function, and relevance to disease. Ann N Y Acad Sci 2008;1124:1-38

35. Zhang Z, Lu G, Zhong Y, et al. Altered spontaneous neuronal activity of the default-mode network in mesial temporal lobe epilepsy. Brain Res 2010;1323:152-60

36. Liao W, Zhang Z, Pan Z, et al. Default mode network abnormalities in mesial temporal lobe epilepsy: a study combining fMRI and DTI. Hum Brain Mapp 2011;32:883-95

37. Zeng H, Pizarro R, Nair VA, et al. Alterations in regional homogeneity of resting-state brain activity in mesial temporal lobe epilepsy. Epilepsia 2013;54:658-66

38. Luo C, Qiu C, Guo Z, et al. Disrupted functional brain connectivity in partial epilepsy: a resting-state fMRI study. PLoS One 2011; 7:e28196

39. Dosenbach NU, Fair DA, Cohen AL, et al. A dual-networks architecture of top-down control. Trends Cogn Sci 2008;12:99-105

40. De Pisapia N, Turatto M, Lin P, et al. Unconscious priming instructions modulate activity in default and executive networks of the human brain. Cereb Cortex 2012;22:639-49

41. Corbetta M, Shulman GL. Control of goal-directed and stimulusdriven attention in the brain. Nat Rev Neurosci 2002;3:201-15

42. Zamarian L, Trinka E, Bonatti E, et al. Executive functions in chronic mesial temporal lobe epilepsy. Epilepsy Res Treat 2011;2011:596174

43. Bell B, Lin JJ, Seidenberg M, et al. The neurobiology of cognitive disorders in temporal lobe epilepsy. Nat Rev Neurol 2011;7:154-64

44. Keller SS, Baker G, Downes JJ, et al. Quantitative MRI of the prefrontal cortex and executive function in patients with temporal lobe epilepsy. Epilepsy Behav 2009;15:186-95

45. Hermann BP, Lin JJ, Jones JE, et al. The emerging architecture of neuropsychological impairment in epilepsy. Neurol Clin 2009;27: 881-907

46. Zuo XN, Ehmke R, Mennes M, et al. Network centrality in the human functional connectome. Cereb Cortex 2012;22:1862-75

47. Fair DA, Cohen AL, Power JD, et al. Functional brain networks develop from a "local to distributed" organization. PLoS Comput Biol 2009;5:e1000381 